Hautarzt $2021 \cdot 72: 561-562$

https://doi.org/10.1007/s00105-021-04840-2

Angenommen: 12. Mai 2021

(c) Springer Medizin Verlag $\mathrm{GmbH}$, ein Teil von Springer Nature 2021

\section{Update - Mikrobiom: vom Hype zur Glaskugel und zum wertvollen Therapiewerkzeug!}

\author{
Claudia Traidl-Hoffmann ${ }^{1,2} \cdot$ Bernhard Homey $^{3} \cdot$ Stephan Weidinger ${ }^{4}$ \\ ${ }^{1}$ Institut für Umweltmedizin, Helmholtz Zentrum München, München, Deutschland \\ ${ }^{2}$ Lehrstuhl für Umweltmedizin, Medizinische Fakultät, Universität Augsburg, Augsburg, Deutschland \\ ${ }^{3}$ Klinik für Dermatologie, Universitätsklinikum Düsseldorf, Medizinische Fakultät, Heinrich-Heine- \\ Universität Düsseldorf, Düsseldorf, Deutschland \\ ${ }^{4}$ Klinik für Dermatologie, Venerologie und Allergologie, Universitäts-Hautklinik Kiel, Universitätsklinikum \\ Schleswig-Holstein, Kiel, Deutschland
}

Uns Hautärzten ist die Bedeutung von Mikroben seit Jahrzehnten bewusst. Wir kennen sie als ursächlich verantwortliche, aber auch als Triggerfaktoren entzündlicher Hauterkrankungen. Mit dem Aufkommen von umfassenden Analysemethoden wie der "Next-Generation-Sequenzierung" haben wir heute eine beispiellose Möglichkeit, nicht nur das menschliche Genom zu durchleuchten, sondern auch das der in und auf unserer Haut lebenden Mikroorganismen. Diese Technologien, die das Feld der "Omics" erst erschließen, können ohne komplexe bioinformatische Werkzeuge und große Rechnerkapazität gar nicht nutzbar gemacht werden; die Analyseergebnisse können ohne sie nicht für die Praxis interpretiert werden. Deswegen ist bis zum heutigen Tag die Mikrobiomanalyse sowohl des Darms als auch der Haut ein Feld der Forschung und in seriösen Händen kein Routinediagnostikwerkzeug. In unseren Patientengesprächen werden uns Ampelergebnisse von Mikrobiomuntersuchungen vorgelegt, auf deren Grundlage wir als Mediziner sinnvolle Diagnosen stellen oder gar Therapieoptionen entwickeln sollen. Allerdings ist das - Stand heute - nicht möglich. Was wir über das Mikrobiom von Grenzflächenorganen wissen, ist zugegeben schon viel - aber noch nicht genug, um daraus Konsequenzen für therapeutische Konsequenzen abzuleiten. Dafür wird noch viel Forschung vonnöten sein.

Die meisten Mikroorganismen des Mikrobioms kommen in Symbiose mit uns als Kommensalen nicht nur gut aus, sondern sind vielmehr durch eine langfristige Anpassung für uns unentbehrlich geworden. Wir bestehen je zur Hälfte aus bakteriellen und menschlichen Zellen [1], wobei das Genom der Bakterien das unsere an genetischer Information um 1 bis 2 10erPotenzen übersteigt. Das Hautmikrobiom gilt heute als aktiver Teil der funktionelIen Hautbarriere [2]. Diese Perspektive auf das Mikrobiom als Schutzfaktor wird durch den ersten Beitrag dieser Ausgabe von Professor Bosch vom Zoologischen Institut der Universität Kiel wissenschaftlich umfassend und gleichzeitig visionär, fast philosophisch diskutiert. Wohl orchestriert setzt sich das Mikrobiom aus unterschiedlich vielen und gleichzeitig personalisierten Mischungen von Mikroben (Viren, Pilze, Bakterien) zusammen. Bosch dreht den Songtitel der Rolling Stones „The singer not the song" um: "It's the song, not the singer", und macht so deutlich, dass dieses Mikrobiomorchester "wohltemperiert" zusammenspielt. Der Zusammenklang macht es aus, nicht das singuläre Bakterium - eben der Song und nicht der Singer. Im gesunden Zustand ist dieser Song im Wohlklang, bei Entzündung eher Zwölftonmusik. Interessant ist, dass trotz der umfassenden Forschung bis heute nicht klar ist, was wirklich ein gesundes Mikrobiom ausmacht. Bosch stellt das Thema Mikrobiom in den globalen Kontext: „Aus unserer evolutionären Perspektive wird deutlich, dass die einzigartig entwickelte Fähigkeit des Menschen, die Umwelt schnell und schneller zu verändern, zunehmend die Funktionalität unseres Mikrobioms außer
Kraft setzt." Sicher sind sich Ärzte und Wissenschaftler bezüglich einer Sache: Eine reduzierte Vielfalt des Mikrobioms geht oft mit Entzündungen einher. Der Verlust von Biodiversität zieht sich wie ein roter Faden durch das Anthropozän, sodass Bosch mahnend äußert: „Nicht der Mensch alleine, sondern das multiorganismische Kollektiv des Holobionten ist der Patient." Er zeigt gleichzeitig auf, dass der Verlust von mikrobieller Vielfalt bei fast allen umweltbedingten nichtkommunizierbaren Erkrankungen (z. B. Krebs, Asthma, Allergien) zu finden ist.

\section{)> Die Mikrobiomanalyse von Darm und Haut ist ein Feld der Forschung und kein Routinediagnostikwerkzeug}

Der Zusammenhang zwischen chronisch entzündeter Haut, immunologischen Reaktionen in der Haut und dem Mikrobiom wird im zweiten Übersichtsbeitrag dargestellt. Kuchner et al. fassen aktuelles Wissen über das Gleichgewicht von Immunsystem und Hautmikrobiom bei der atopischen Dermatitis und der Psoriasis vulgaris zusammen. Hierbei wird erneut deutlich, wie stark Mikrobiom und Transkriptom zusammenhängen [3]. Moitinho-Silva et al. bestätigten erst kürzlich, dass Umwelt und Lebensgewohnheiten zwar eine Rolle spielen, jedoch auch die Mikroumgebung der Haut und die Wirtsphysiologie einen sehr starken Einfluss auf die Mikrobiomzusammensetzung der Haut haben [4]. Diese und viele andere Erkenntnisse haben dazu ge- 
führt, dass bei der atopischen Dermatitis bereits topische Anwendungen zur direkten Beeinflussung des Mikrobioms in Erprobung sind, wobei bei der Psoriasis noch weitere Erkenntnisgewinne bezüglich der pathophysiologischen Bedeutung der Mikrobiota notwendig sind. Zu klären bleibt, ob der Verlust von Diversität bei der atopischen Dermatitis Henne oder Ei ist. Bisherige Daten weisen auf eine Doppelrolle hin - also sowohl Henne als auch Ei.

Im abschließenden Beitrag von Hülpüsch und Reiger erfolgt eine kurze Darstellung zur Frage, ob das Hautmikrobiom ein diagnostisches und therapeutisches Ziel im Kontext chronisch entzündlicher Hauterkrankungen darstellen könnte. Dabei wird klar, dass bei der Neurodermitis der übliche Verdächtige Staphylococcus aureus nicht nur pathogenetisch eine zentrale Rolle spielt und somit erklärter Angriffspunkt zielgerichteter Mikrobiomtherapien sein sollte, sondern sich auch als Biomarker anbietet. Staphylococcus aureus ist ein Prädiktor für den Krankheitsverlauf einer Neurodermitis und hat sogar Vorhersagewert für das langfristige Ansprechen eines Patienten auf Systemtherapien [5].

Die Mikrobiomforschung startete im Darm und verfügt hier bereits über klare therapeutische Implikationen. Die Hautmikrobiomforschung hinkt(e) lange hinterher, insbesondere aufgrund der vielen methodischen Herausforderungen, die zum großen Teil durch die geringen Materialvolumina bedingt sind [6]. Deswegen ist die Hautmikrobiomforschung immer noch methodisch komplex und anfällig für Kontaminationen und somit Fehlinterpretationen. Auf der anderen Seite erfreut sich die Hautmikrobiomforschung einer hohen anatomischen Genauigkeit. Die Darmmikrobiomforschung ist bis heute eine Stuhlmikrobiomforschung. An der Haut können wir lokal genau hinschauen - und deshalb dann auch lokal genau intervenieren. Wir sind davon überzeugt, dass das Hautmikrobiom Ziel therapeutischer, maßgeschneiderter Interventionen sein wird und gleichzeitig als Biomarker und diagnostisches Werkzeug zur Verfügung stehen wird. Autoren dieser Ausgabe werden hierzu in der Zukunft wichtige Beiträge leisten. Einen Überblick zum jetzigen Stand der Forschungen bieten sie heute für unsere Leser.
Korrespondenzadresse

Prof. Dr. Claudia Traidl-Hoffmann

Lehrstuhl für Umweltmedizin, Medizinische Fakultät, Universität Augsburg

Neusässer Str. 47, 86156 Augsburg, Deutschland

claudia.traidl-hoffmann@med.uniaugsburg.de

Univ.-Prof. Dr. Bernhard Homey

Klinik für Dermatologie, Universitätsklinikum Düsseldorf, Medizinische Fakultät, HeinrichHeine-Universität Düsseldorf Moorenstr. 5, 40225 Düsseldorf, Deutschland Bernhard.Homey@med.uni-duesseldorf.de

\section{Prof. Dr. Stephan Weidinger}

Klinik für Dermatologie, Venerologie und Allergologie, Universitäts-Hautklinik Kiel, Universitätsklinikum Schleswig-Holstein Arnold-Heller-Str. 3, 24105 Kiel, Deutschland sweidinger@dermatology.uni-kiel.de

Interessenkonflikt. C. Traidl-Hoffmann war als Beraterin für die Firmen Bencard Allergy, Novartis und Eli Lilly tätig und hat Vorträge für La Roche Posay, Galderma, Sebapharma, Novartis und Sanofi Genzyme gehalten. B. Homey war als Berater für die Firmen Novartis, Eli Lilly, Leo Pharma, Galderma, AbbVie, Pfizer, Union Therapeutics tätig und hat Vorträge für Novartis, Eli Lilly, Leo Pharma, Galderma, AbbVie, Pfizer gehalten. S. Weidinger war als Berater für die Firmen Sanofi Genzyme, Regeneron, Leo Pharma, AbbVie, Eli Lilly, Pfizer, Galderma, Kymab, GSK tätig und hat Vorträge für Sanofi Genzyme, Regeneron, Leo Pharma, AbbVie, Eli Lilly, Pfizer gehalten.

\section{Literatur}

1. Sender R, Fuchs S, Milo R (2016) Are we really vastly outnumbered? Revisiting the ratio of bacterial to host cells in humans. Cell 164(3):337-340

2. Eyerich $S$ et al (2018) Cutaneous barriers and skin immunity: differentiating a connected network. Trends Immunol 39(4):315-327

3. Ottman N et al (2021) Microbial and transcriptional differences elucidate atopic dermatitis heterogeneity across skin sites. Allergy 76(4):1173-1187

4. Moitinho-Silva L et al (2021) Host traits, lifestyle and environment are associated with the human skin bacteria. Br J Dermatol. https://doi.org/10. 1111/bjd.20072

5. Hulpusch C et al (2020) Skin pH-dependent staphylococcus aureus abundance as predictor for increasing atopic dermatitis severity. Allergy 75(11):2888-2898

6. Kong HH et al (2017) Performing skin microbiome research: a method to the madness. J Invest Dermatol 137(3):561-568

\section{Ausgabe verpasst?}

Jetzt als ePaper lesen!

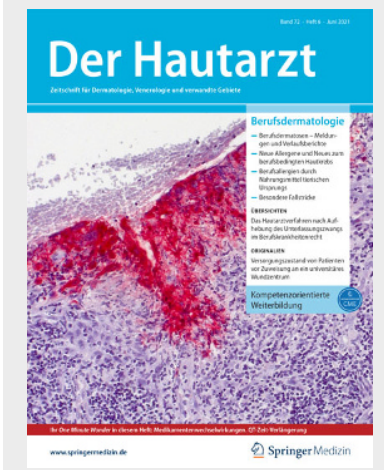

Lesen Sie die Zeitschrift Der Hautarzt jetzt auch digital auf Ihrem Tablet oder Smartphone - jederzeit und überall. SpringerMedizin.de hält für Sie alle Ausgaben der letzten drei Jahre als ePaper bereit, auf die Sie kostenfrei zugreifen können.

Highlights der Juniausgabe zum Thema Berufsdermatologie:

- Ärztliche Meldung von

Berufskrankheiten in der dermatologischen Praxis

- Neue Allergene

- Neues zum berufsbedigten Hautkrebs

- Außergewöhnliche Berufsallergien durch Nahrungsmittel tierischen Ursprungs

- Besondere Fallstricke

- Das Hautarztverfahren nach Aufhebung des Unterlassungszwangs im Berufskrankheitenrecht

- Die neue Weiterbildungsordnung Dermatologie: Neuer Wein in alten Schläuchen?

- CME: Kompetenzorientierte Weiterbildung

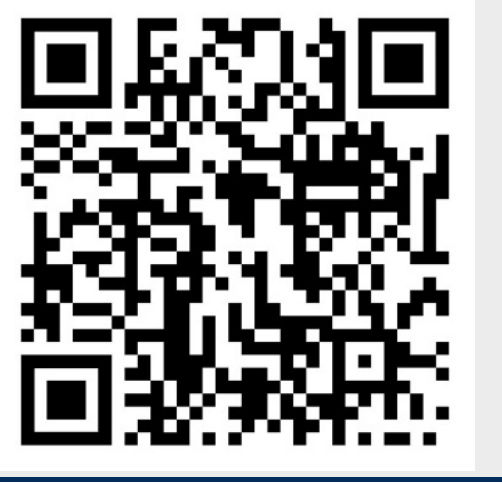

